\title{
Effects of beverage carbonation on lubrication mechanisms and mouthfeel
}

Sorin-Cristian Vlădescu ${ }^{\mathrm{a}}$, Sophie Bozorgi ${ }^{\mathrm{b}}$, Songtao Hu ${ }^{\mathrm{a}, \mathrm{c}}$, Stefan K. Baier ${ }^{\mathrm{d}, \mathrm{e}}$, Connor Myant ${ }^{\dagger}$, Guy Carpenter ${ }^{b}$, Tom Reddyhoffa

a Tribology Group, Department of Mechanical Engineering, Imperial College London, South Kensington, Exhibition Road, SW7 2AZ, London, United Kingdom

b Salivary Research Unit, Faculty of Dental, Oral and Craniofacial Sciences, King's College London Dental Institute, Floor 17 Guy's Tower, London, SE1 9RT, UK

c State Key Laboratory of Mechanical System and Vibration, Shanghai Jiao Tong University, Shanghai 200240, China

d PepsiCo Global Functions, Measurement Science, 3 Skyline Drive, Hawthorne, NY 10532, U.S.A.

e School of Chemical Engineering, The University of Queensland, Brisbane 4072, Queensland, Australia

${ }^{f}$ Robotics and Manufacturing Group, Dyson School of Design Engineering, Imperial College London, London, SW7 2AZ, United Kingdom

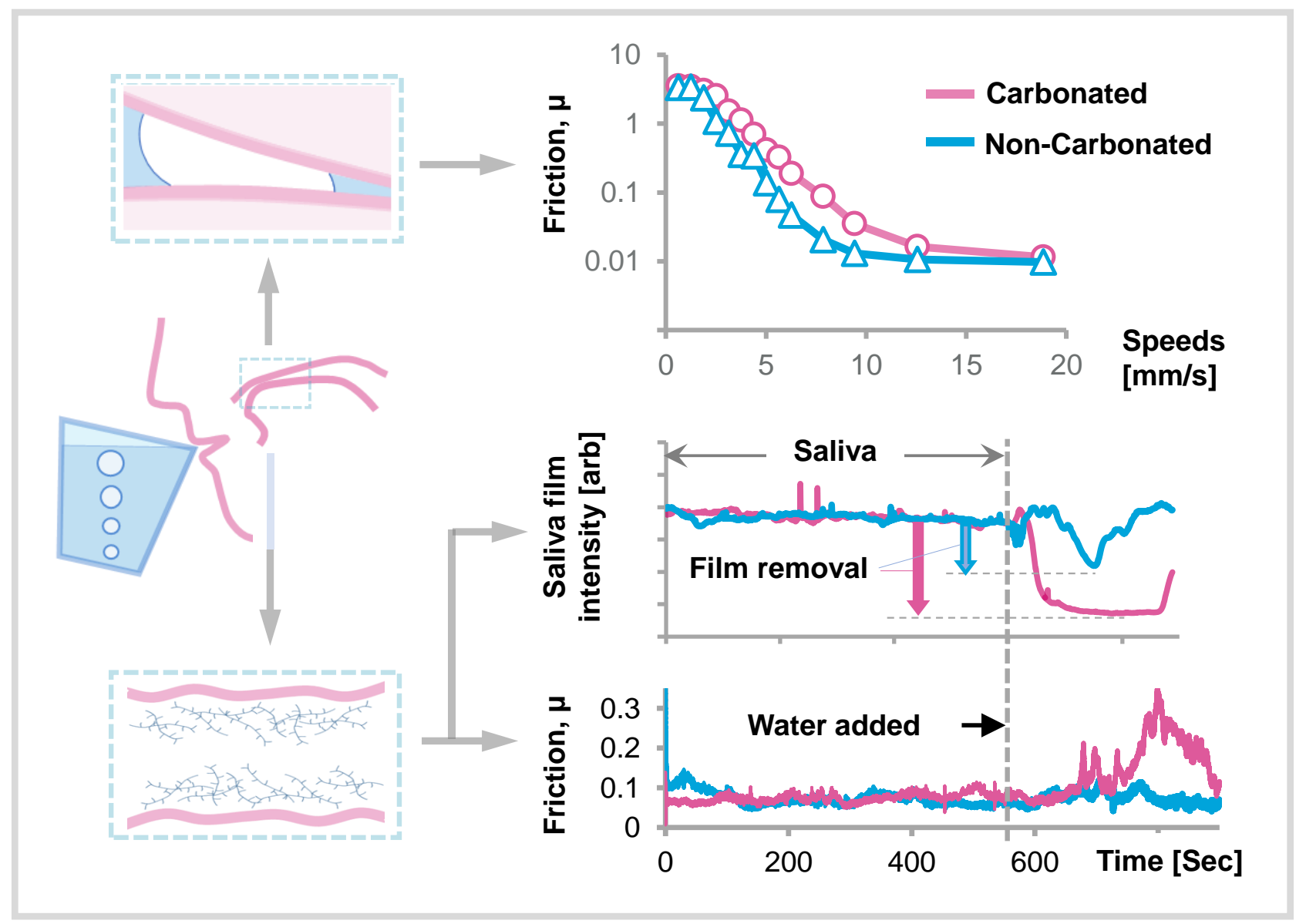

FIGURE 0 - Graphical Abstract 


\begin{abstract}
The perception of carbonation is an important factor in beverage consumption which must be understood in order to develop healthier products. Herein, we study the effects of carbonated water on oral lubrication mechanisms involved in beverage mouthfeel and hence taste perception. Friction was measured in a compliant PDMS-glass contact simulating the tongue-palate interface (under representative speeds and loads), while fluorescence microscopy was used to visualise both the flow of liquid and oral mucosal pellicle coverage.

When carbonated water is entrained into the contact, $\mathrm{CO}_{2}$ cavities form at the inlet, which limit flow and thus reduce the hydrodynamic pressure. Under mixed lubrication conditions, when the fluid film thickness is comparable to the surface roughness, this pressure reduction results in significant increases in friction (>300\% greater than under non-carbonated water conditions). Carbonated water is also shown to be more effective than non-carbonated water at debonding the highly lubricious, oral mucosal pellicle, which again results in a significant increase in friction. Both these transient mechanisms of starvation and salivary pellicle removal will modulate the flow of tastants to taste buds and are suggested to be important in the experience of taste and refreshment. For example this may be one reason why flat colas taste sweeter.
\end{abstract}

Keywords: Carbonation, Beverages, Bubble growth, Salivary lubrication, Fluorescence imaging, Mouthfeel, Friction, Oral Tribology. 


\section{Introduction}

Carbonated beverages such as soft drinks, beer and sparkling wine are hugely popular. In the US alone, it is estimated that $\$ 80.6$ billion worth of carbonated products were sold in 2016 (Beverage Digest, 2017).

The sensory effects of carbonation are subtle and varied [1]. Carbonation can enhance certain flavours, such as sourness and saltiness, and inhibit others [2]. Carbonation can mask mouthfeel differences between diet and regular colas [3]. However, too low or too high carbonation results in an overall imbalance of flavours [4]. The formation and growth of $\mathrm{CO}_{2}$ bubbles in particular can have a considerable effect on flavour release and taste perception [5] as demonstrated, for instance, by bubble size playing a key role in the assessment of a Champagne wine [6]. The application of these effects in the development of products is limited by a lack of knowledge of the underlying physics.

Carbonated beverages are widely regarded as eliciting a pleasurable taste despite the sensation of ingesting a carbonated drink being irritating or painful $[7,8]$. Research on mammals has shown carbonation can provoke both somatosensory and chemosensory responses, as well as activate taste neurons. Supporters of the mechanical origin of carbonated liquid sensation argued this is caused by bursting $\mathrm{CO}_{2}$ bubbles activating mechanoreceptors [9]. Multiple lines of research have more recently provided supporting evidence in favour of the chemogenic origin of carbonation sensation. In a series of papers, Carsterns et al. [10-12] provided psychophysical and neuroanatomical support for this hypothesis, concluding that carbonated water stimulates polymodal nociceptors on the tongue through the conversion of $\mathrm{CO}_{2}$ into carbonic acid. The nociceptors then excite the trigeminal neurons which signal oral irritation to the brain. More recently, Zuker et al. [13] conducted a study to understand taste receptors' role in detecting and signalling carbonation, outlining the cellular and molecular substrates responsible for this taste. They used engineered mice, in which different populations of receptor cells were selectively ablated by targeted expression of attenuated diphtheria toxin and identified the sour-sensing cells as responsible for detecting carbonation. It was also shown that carbonic anhydrase 4, a glycosylphosphatidylinositol-anchored enzyme, functions as the primary $\mathrm{CO}_{2}$ taste sensor. The current understanding is that the "fizz" generated by carbonated beverages is the result of a combination of somatosensory system stimulation $[10-12,14]$ and the activation of the sour-sensing taste buds.

When consumers drink a carbonated beverage, they not only taste the product but their saliva also interacts with the product. It has been suggested that somatosensory and chemosensory processes are not solely responsible for the drinking experience and that the tribological behaviour of liquids also plays a significant role $[15,16]$. Tribology is the study of friction and lubrication between interacting surfaces in relative motion, and tribological phenomena accompany all food and beverage consumption as these involve various sliding 
surfaces in contact, for example tongue-food, tongue-bolus and food particles-oral surfaces. An in-depth description of tribology fundamentals as they relate to food lubrication can be found in a recent review on 'oral tribology' [17], whereas [18] details the development of tribology as a contributing discipline for understanding oral processing and the rheological properties of food.

Mouth friction is dominated by the effects of easily deformable components combined with thin films of surface-active species within saliva, a highly complex mixture of proteins with non-Newtonian, viscoelastic behaviour which also binds to the mucosa to form a hydrogel interface [19]. Saliva's key role is to lubricate the oral cavity and protect mucous membranes from damage by allowing food to be chewed and swallowed without effort; those who suffer from xerostomia (dry mouth) can attest that the lack of saliva is a highly unpleasant condition [20].

We hypothesise that the popularity of a carbonated beverage is determined not only by its rheological and chemical properties, but also by those which determine how it combines and interacts with in-mouth saliva lubrication mechanisms. In this paper, we demonstrate that both the hydrodynamic and the boundary lubricating components of the salivary film are reduced by the interaction of oral surfaces with $\mathrm{CO}_{2}$ bubbles. To understand this behaviour, we developed a method of visualising the salivary film and measuring the friction force in a tribological contact lubricated with whole mouth saliva (WMS), allowing both qualitative and quantitative assessments of the impact of carbonated water on salivary film. This utilizes an existing test protocol developed to accurately mimic the sliding of oral surfaces during beverage consumption [21]. This research complements previous studies of bubble growth in carbonated liquids which were carried out on liquids under static conditions $[5,22,23]$. 


\section{Methods and Materials}

\subsection{Methods}

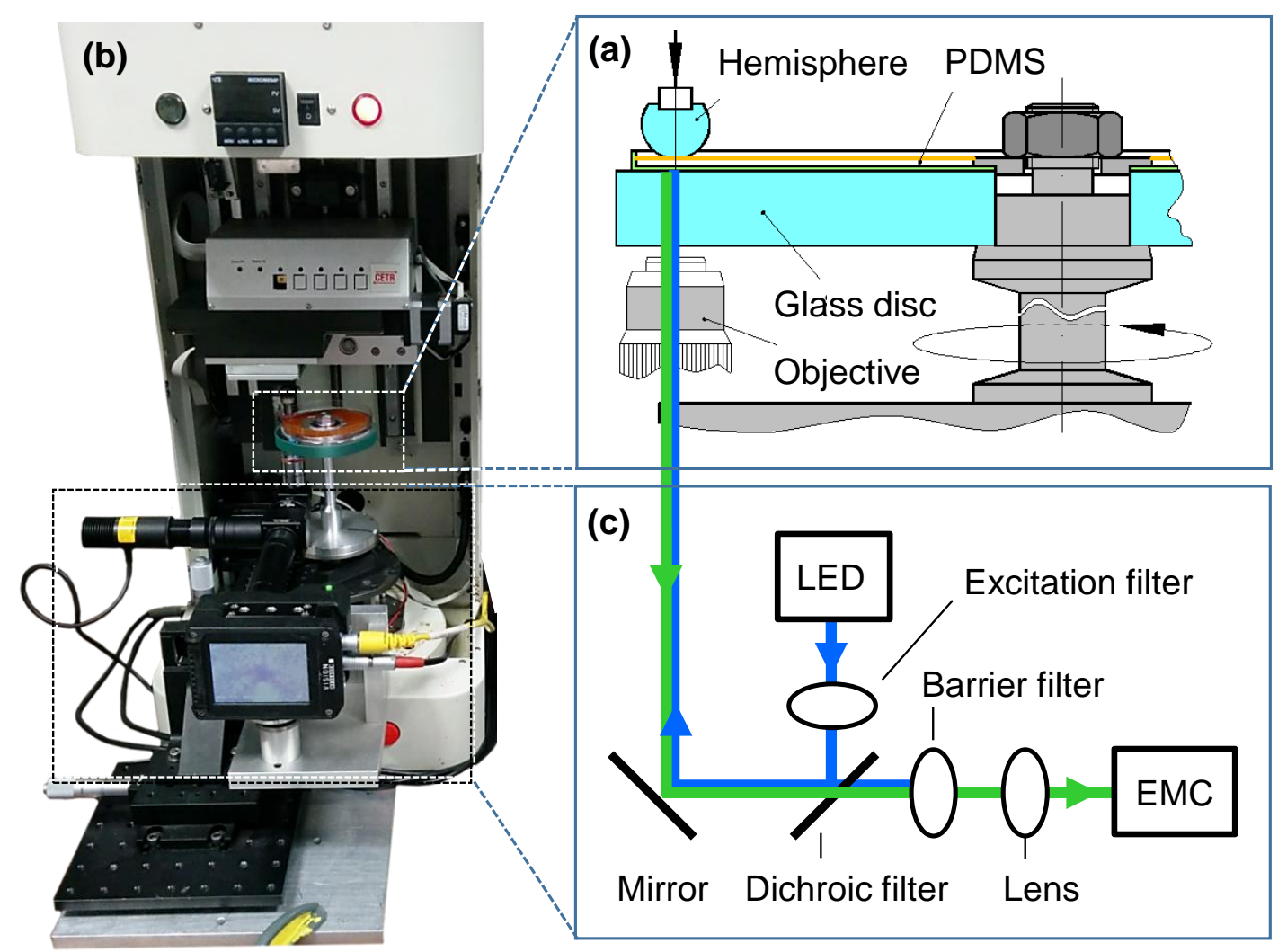

FIGURE 1 - (a) Schematic representation of the oral mimic visualisation setup; (b) photograph of modified CETR UMT2 tribometer; (c) schematic of fluorescent microscope set up.
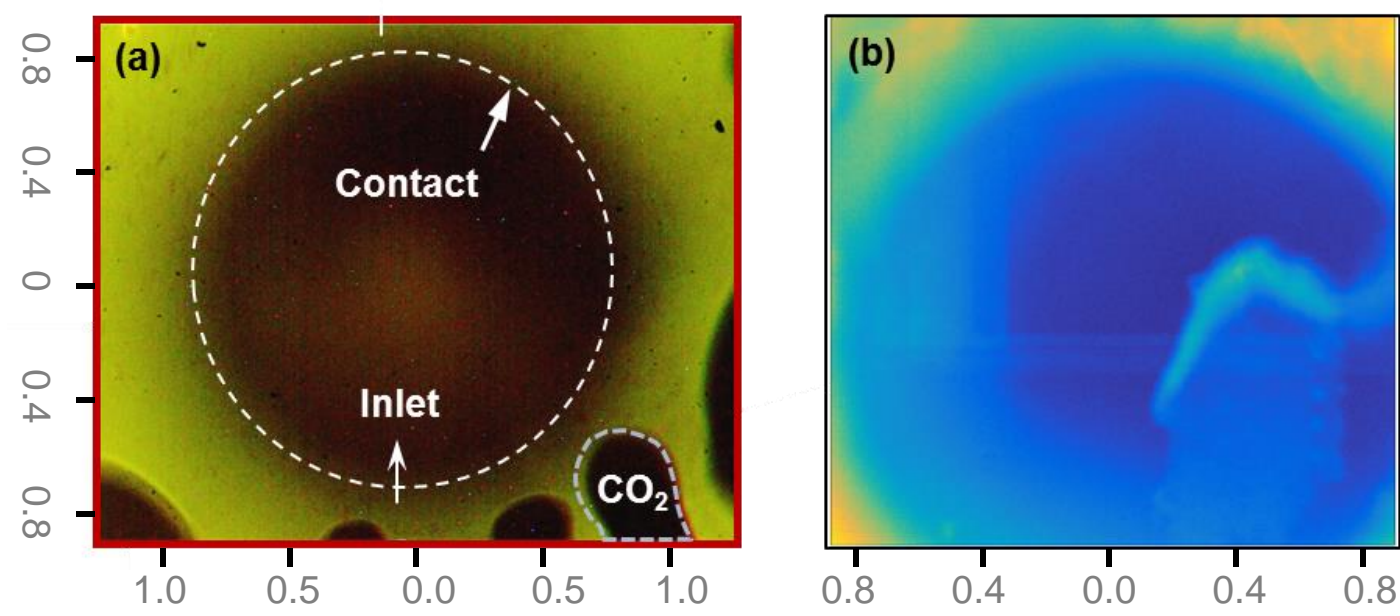

$\operatorname{Max} I$

FIGURE 2 - Fluorescent images of the contact between silica hemisphere and PDMS specimen lubricated by: (a) FITC dyed carbonated water, (b) FITC tagged salivary proteins. 
In this study, we used a previously developed test set-up and protocol to assess beverages under lubrication conditions that mimic those found within the mouth [21]. This involved loading a $5 \mathrm{~mm}$ radius silica hemisphere against a rotating polydimethylsiloxane (PDMS) disc that was fixed onto a transparent support (Figure 1a). These elements were located in a UMT-2 tribometer (Universal Materials Tester from Bruker), (Figure 1b). The equipment was operated in pin-on-disc mode, so that the PDMS specimen rotated while the silica hemisphere was held stationary. The setup enables a range of sliding speeds, similar to those encountered in the tongue-palate contact $(0.5$ to $20 \mathrm{~mm} / \mathrm{s})$, while accurately controlling and measuring the normal load and friction force using sensitive strain gauges, bonded to the housing above the stationary silica hemisphere specimen.

A custom-built, Laser Induced Fluorescence (LIF) microscope was positioned beneath the sliding contact and focussed through the transparent PDMS and supporting disc and onto the salivary film (Figure 1c). When a dyed liquid is present in or around the contact interface, it becomes excited by incident light from the microscope's LED and fluoresces. The incident and emitted light, of different wavelengths, are separated by a dichroic filter and the resulting images are captured by an intensified camera. For film thicknesses greater than $200 \mathrm{~nm}$, the intensity of the fluorescence light emitted from the contact is proportional to the thickness of the liquid in the interface. This means that the images acquired by the camera represent maps showing the thickness of dyed liquid in the contact. Further details of LIF technique can be found in [24-26].

\subsection{Materials}

Initially it was necessary to image the distribution of water/ $\mathrm{CO}_{2}$ around a contact lubricated with carbonated water (without saliva). This was achieved using carbonated deionised water as lubricant, into which the dye fluorescein isothiocyanate (FITC, $\geq 90 \%$ (HPLC), powder, Sigma-Aldrich) was dissolved at a concentration of $0.1 \mathrm{~mol} / \mathrm{l}$. A resulting fluorescence image captured under transient conditions is shown in Figure 2a. Here, dark chartreuse regions indicate the presence of deionised water, while black regions indicated carbonation bubbles (no liquid). In the figure, the sliding direction of the PDMS specimen, relative to the stationary silica hemisphere specimen, is indicated with white arrows, together with the contact's inlet and outlet. The contact area is highlighted by the region enclosed by the dotted yellow circle.

In the second set of tests, visualisation of saliva interactions within the contact was enabled by using individually dye-tagged saliva proteins (FITC). Note: saliva was collected from a self-reported healthy subject by passively drooling into a tube over 10 minutes. The sample was kept on ice until further processing. For FITC-staining of salivary proteins, $1 \mathrm{ml}$ of saliva $(1.8 \mathrm{mg} / \mathrm{ml}$ total protein) was mixed with $1 \mathrm{ml}$ of a FITC solution $(5 \mathrm{mg} / 100 \mathrm{ml}$ carbonate buffer pH9.6) for one hour in the dark with constant mixing. The solution was then dialysed extensively against PBS until the eluate was non-fluorescent. A fluorescence image captured 
during a tagged saliva test is shown in Figure $2 \mathrm{~b}$. Here the bright regions within the contact show adhered protein boundary film.

The silicon-based organic polymer, Polydimethylsiloxane (PDMS), was used for the oral mimic surface since, as we have previously shown [21], it binds salivary proteins and shows similar friction characteristics to porcine tongue samples when lubricated by an aqueous salivary solution. Furthermore, this polymer has known surface chemistry and is highly homogeneous, in contrast to actual biological samples [15]. Due to its repeating $-\mathrm{O}-\mathrm{Si}\left(\mathrm{CH}_{3}\right)_{2-}$ group, PDMS is hydrophobic and attracts proteins indiscriminately [27] (the adherence of proteins to PDMS is a problematic occurrence in lab-on-chip systems [27]). PDMS is also well defined mechanically, having been used previously in numerous tribological studies (e.g. [28-30]). Our PDMS specimens were moulded using a commercially available Sylgard 184 kit from Dow-Corning. The upper hemispherical specimen is glass, which is harder than the oral palate which it represents. However, this is not expected to effect the lubrication behaviour of the contact as the entrainment of fluid is dependent on the composite stiffness of the contact which in turn is dominated by the lower of the two stiffnesses (i.e. that of the PDMS).

\section{Results and discussion}

We first considered the sweetness perception responses of nine subjects through VAS scores after consuming carbonated and non-carbonated cola (Figure 3 - detail). In agreement with previous findings, carbonation results in a significant decrease in sweetness perception. Current understanding is that this is due to $\mathrm{CO}_{2}$ bubbles eliciting a distraction to the brain, since their conversion into carbonic acid triggering an irritative response via the tongue's nociceptors. However, we also conducted PDMS sliding tests, measuring frictional response of model carbonated and non-carbonated sucrose solutions mixed with saliva (Figure 3), which showed that carbonation also has a marked effect on friction. As detailed below and in [21], the combination of conditions (stiffness, roughness, geometry) for the test shown in Figure 3 is such that the contact transitions from boundary (intimate surface contact) to mixed to full-film (fully separated surfaces) lubrication regimes as the sliding speed is increased up to $20 \mathrm{~mm} / \mathrm{s}$. Despite this, friction is approximately constant with sliding speed. This is because the surface-active salivary proteins reduce the boundary and mixed lubrication friction in the contact bringing so there is only a very slight reduction in friction as the contact transitions into full-film lubrication. 


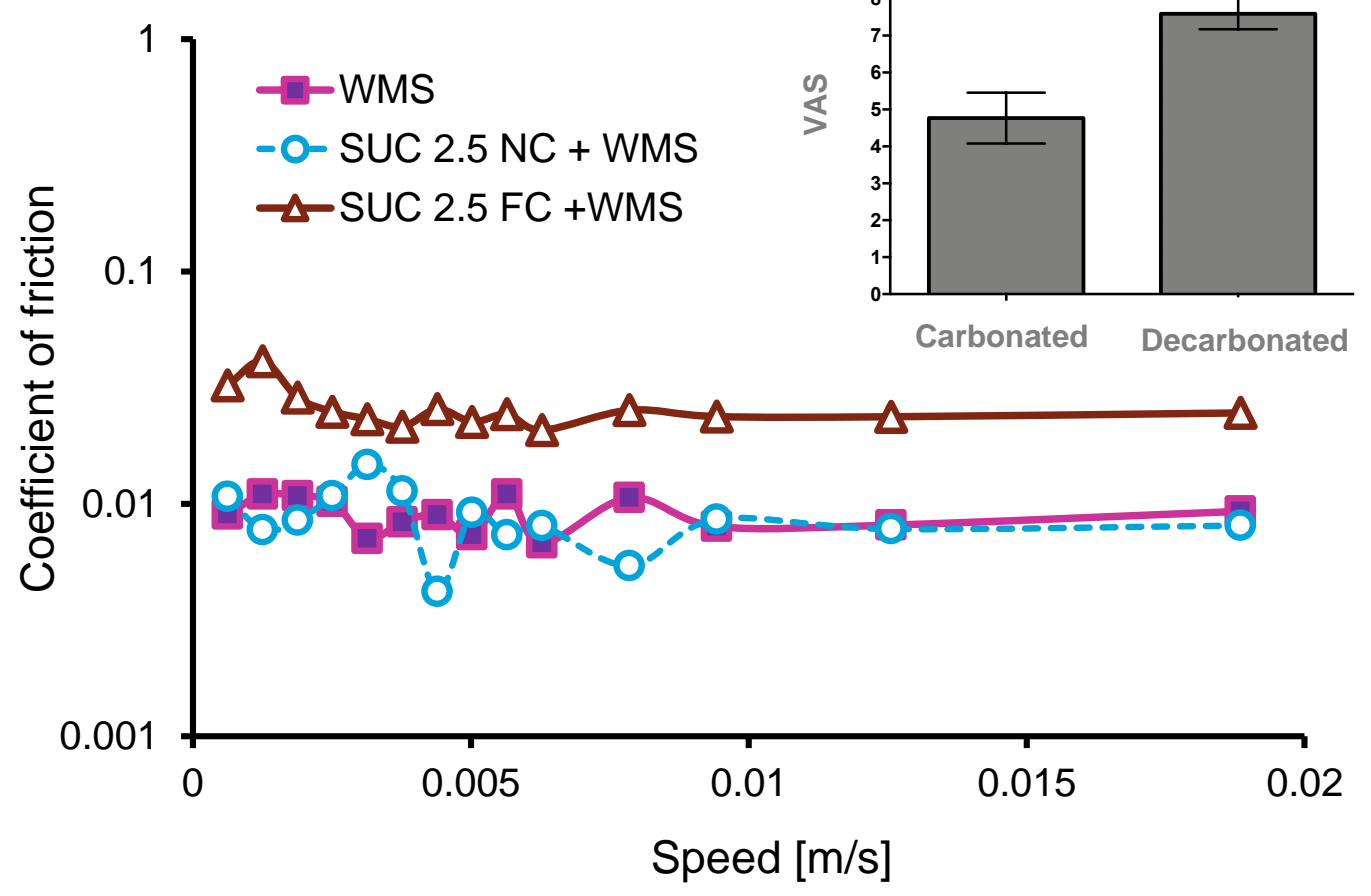

FIGURE 3 - Friction coefficient traces as recorded for carbonated and non-carbonated mixtures of sucrose and saliva (Insert: VAS score of nine subjects, $p<0.005)$ with an applied load of $0.2 \mathrm{~N}$. Legend: $W M S=$ Whole Mouth Saliva, SUC $=$ Sucrose, FC = Fully Carbonated, $N C=$ Non-Carbonated.

To investigate the difference in friction between the carbonated and non-carbonated solutions, two sets of tests were run: i) Stribeck curves (varying speed while measuring the variation in friction) to assess the impact of carbonation on liquid entrainment - these friction tests were performed simultaneously with fluorescence tests to help understand bubble nucleation and growth, and ii) fluorescence imaging tests to visually monitor the in-contact build-up of a dye-tagged salivary protein film and its subsequent breakdown following the introduction of carbonated and non-carbonated beverages into the contact.

\subsection{Mechanism 1: Inlet Starvation}

Figure 4 shows the friction versus speed behaviour of the contact lubricated by carbonated and non-carbonated, deionised water (note: no saliva films are present during these tests). The two Stribeck curves (varying speed while measuring the variation in friction) were obtained by loading the contact with $0.2 \mathrm{~N}$ and measuring friction while increasing speed from 0.6 to $18.8 \mathrm{~mm} / \mathrm{s}$ (speeds which are typical in oral processing). As speed increases, more liquid is dragged by the surfaces into the contact generating a pressure to separate the two 
components (hydrodynamic lubrication). Thus Stribeck curves give an indication of how friction changes with film thickness and display the following regions. At low speeds, the pressure generated by this hydrodynamic action is insufficient to separate the components (boundary lubrication regime) and friction is dominated by surface effects and in this case is high due to the adhesion between PDMS and glass. As speed increases, friction decreases as components are pushed apart by hydrodynamic pressure so that surface contacts are partially replaced by easily sheared fluid film regions (mixed lubrication). At higher speeds, once surfaces are fully separated, there is no further friction reduction with speed. And, at still higher speeds (though not reached in Figure 4), friction increases as fluid shear becomes the dominant dissipation mechanism. It can be observed in Figure 4 that the carbonated water produces significantly higher friction in the mixed lubrication regime only. This must be attributed to a reduction in water film thickness causing an increase in surface contact - it can neither be due to a) an increase in surface adhesion, since at low-speed boundary friction is the same for the two liquids, nor b) an increase in fluid friction, since the high-speed friction is largely unaffected.

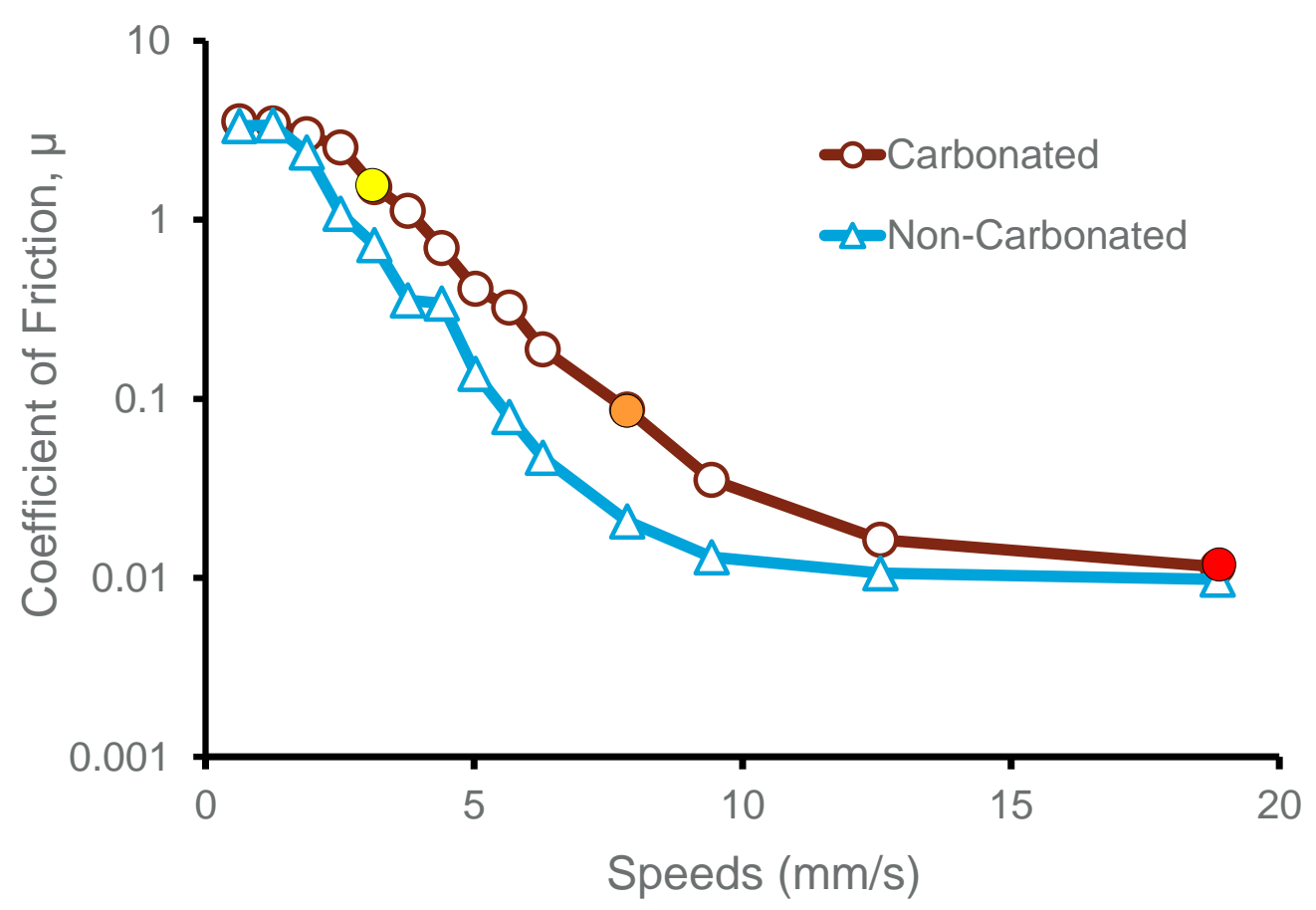

FIGURE 4 - Friction vs. speed curve for contact lubricated with deionised water (no saliva present). Note: the variation between repeated Stribeck curve tests is better that $5 \%$.

To understand and quantify these effects, the LIF system was employed to probe the contact interface. The videos of the contact obtained at the three speeds indicated by coloured markers in Figure 4 were analysed using a Matlab program, which ran through each frame and calculated the percentage of the contact inlet width that was made up of $\mathrm{CO}_{2}$ bubbles. Averaged over the duration of each friction measurement, the percentage of inlet with $\mathrm{CO}_{2}$ is plotted against the increase in friction due to carbonation $\left(\left(\mu_{\text {carb }}-\mu_{\text {non-carb }}\right) / \mu_{\text {non-carb }}\right)$ at each of the speeds in Figure 5. Here, the percentage increase in friction is proportional to the 
fraction of the inlet that is deprived of water. The example images presented in Figure 5 were recorded at the three different sliding speeds (i.e. $3.1,7.8$ and $18.9 \mathrm{~mm} / \mathrm{s}$ ) indicated by coloured markers in Figure 4. The change in order of the measurement points between these two graphs can be noted from the markers' colour. It is observed that, in the mixed regime where friction is affected by carbonation, a large proportion of the water in front of the contact inlet has been displaced by $\mathrm{CO}_{2}$. These results suggest that the observed increase in friction is due to $\mathrm{CO}_{2}$ bubbles starving the contact of water.

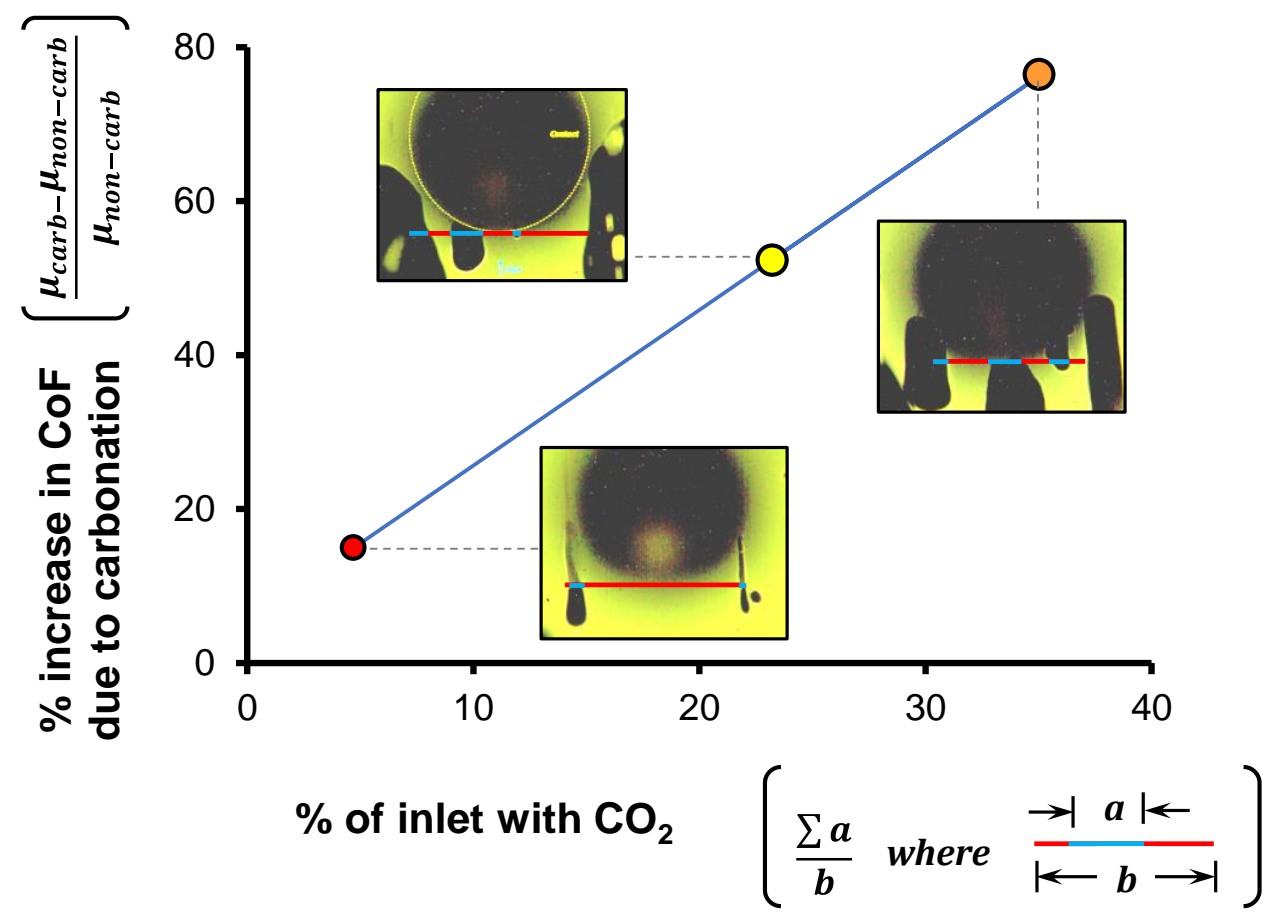

FIGURE 5 - \% increase in friction (comparing $\mu_{\text {carb, }}$ non-carbonated, $\mu_{\text {non-carb, friction data }}$ from Figure 4) vs. \% of inlet that is starved of water (obtained from LIF video images).

The LIF microscopy videos were further analysed to elucidate the mechanisms associated with the increased friction from $\mathrm{CO}_{2}$ induced starvation. Figure 6 shows a selection of video frames (see whole video in Supporting Information) taken from the $7.8 \mathrm{~mm} / \mathrm{s}$ sliding speed test (part of the raw data corresponding to the orange data point in Figure 5). Reviewing the data in figure 6 and $>10$ other bubble entrainments, enable the following observations to be made:

i) Bubbles appear to nucleate at the contact inlet, but do not enter or extend into the contact - those in front of the inlet (e.g. bubble $B$ ) are separated from it by a meniscus. 
ii) Even the fastest moving bubble moves less than $1 \mathrm{~mm}$ between frames. However, in this time the PDMS surface moves $7.8 \mathrm{~mm} / \mathrm{s} \times 0.4 \mathrm{~s}=3.12 \mathrm{~mm}$. Therefore, the upper contact line of each bubble must tend to adhere to the stationary glass specimen while the lower contact line slides continuously against the PDMS surface.

iii) Over time, bubbles increase in size and/or length. They also coalesce.

iv) a) Bubbles in the contact path (i.e. upstream of the inlet) both translate and expand in the upstream direction.

b) Bubbles outside of the contact path translate downstream and simultaneously elongate (elongation occurs until the upstream edge of the bubble reaches the minimum film thickness).

v) Once a bubble outside the contact path (e.g. bubble $A$ ) passes the minimum gap thickness in flow direction, it flows rapidly downstream.

Due to the number of simultaneously occurring mechanisms, a quantitative model of bubble dynamics is beyond the scope of this study. Instead, the following discussion attempts to explain the observations and gain insights into bubble behaviour within the mouth. For this, it is first necessary to detail the lubrication conditions in the experiments with reference to the Figure 7 showing a bubble located upstream of the contact. The bubble is bounded by the stationary hemispherical glass specimen above and the sliding, initially flat, deformed PDMS below. Due to the counter-formal geometry of the two specimens, the overall gap between them converges and then diverges in the direction of flow. As liquid is entrained into this gap due to the sliding motion of the PDMS, a hydrodynamic pressure is generated, which acts to separate the surfaces in the contact (i.e. since the hydrodynamic pressure decreases with gap thickness, the latter adjusts so that the applied load of $0.2 \mathrm{~N}$ is supported). Using Esfahanian and Hamrock's isoviscous-elastohydrodynamic equation [31], the film thickness in the contact area is calculated to be $25 \mathrm{~nm}$ (see details in Supporting Information 2.1), which is comparable to the PDMS surface roughness of $\sim 20$ $\mathrm{nm}$ (this agrees with the data in Figure 4 placing the $7.8 \mathrm{~mm} / \mathrm{s}$ data point in the mixed friction regime). The fluid pressure in the contact results from an interaction between the hydrodynamic flow of liquid and the elastic deformation of the PDMS and could be found by numerically solving Reynolds equation in combination constitutive equations [32]. However, using Hertz contact equations as a first approximation, the maximum and average contact pressure in the contact area are estimated to be 157 and $105 \mathrm{kPa}$ respectively (see detailed calculations in Supporting Information 2.2). Classical Hertz contact mechanics can also be used to predict the deformation of the two components. The results of these film thickness and surface deformation calculations are plotted in Figure 7, along with fluorescence intensity profile (taken from the dashed line in Figure $6 \mathrm{~g}$ ) and thus provides a to-scale representation of the bubble geometry. This shows that the gap between the sliding components reduces by three orders of magnitude between the location of the bubble and the central region of the contact where friction is determined. Outside the pressurised 
region, Couette flow predominates due to the relative motion of the boundary surfaces. Poiseuille flow occurs in the contact due to the converging geometry, however Couette flow still dominates. The capillary number, $\mathrm{Ca}$, (i.e. the ratio of viscous forces to surface tension forces, viscosity $\times$ velocity/surface tension), is calculated to be $\sim 1.08 \times 10^{-4}$, and being significantly less than unity suggests surface tension forces dominate over viscous forces in determining the shape and motion of the bubble, which therefore behaves like a particle [33]. Furthermore, the Bond number, Bo, (i.e. the ratio of gravitational forces to surface tension forces, $\Delta \rho g L^{2} / \gamma$, where $\Delta \rho$ is the difference in density between the gas and liquid phase, and $L$ is the characteristic length - assumed here to be the gap thickness) calculated to be $>5 \times 10^{-4}$, and being significantly less than unity suggests gravitational forces can be ignored.
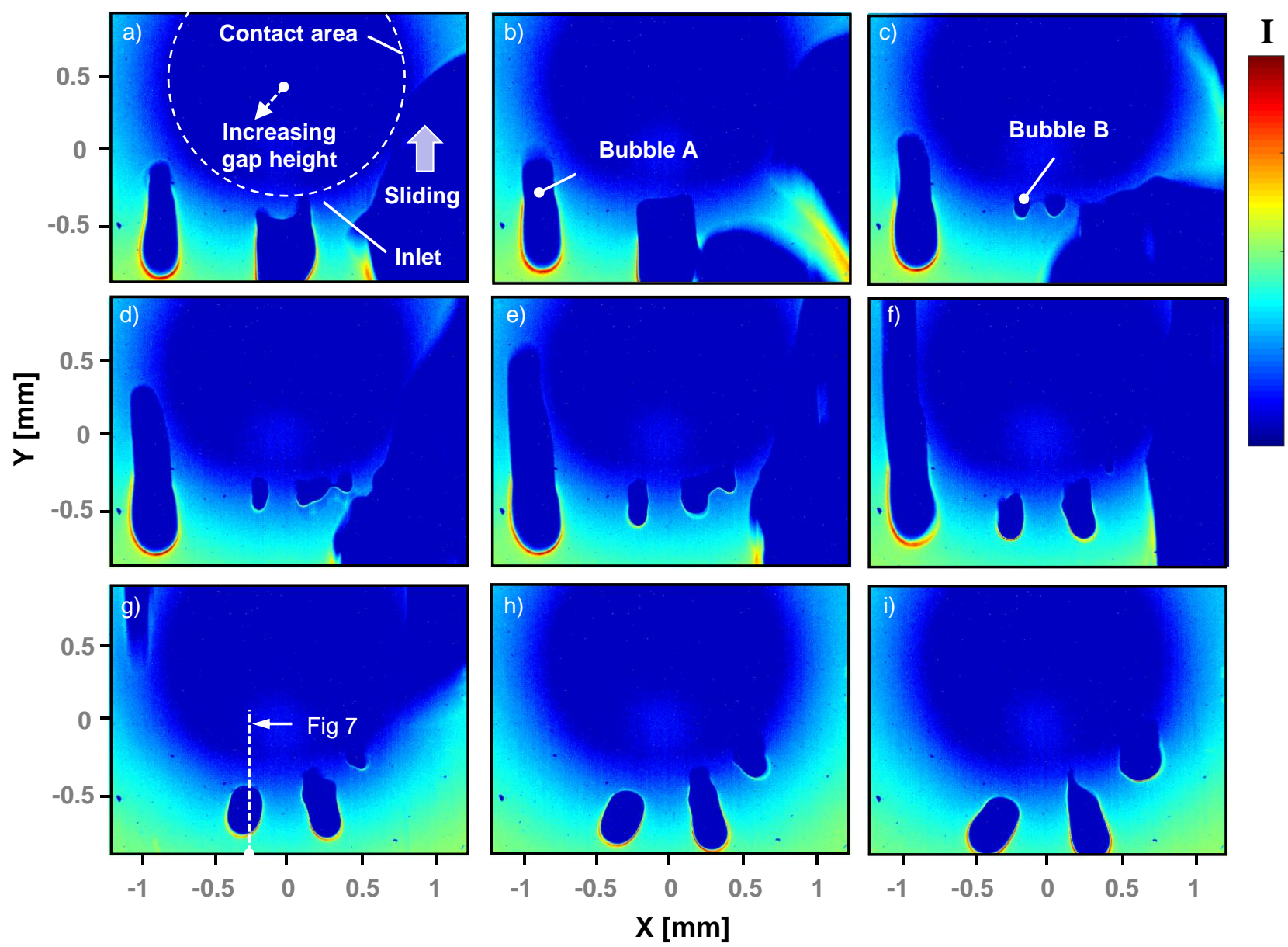

FIGURE 6 - Video frames from LIF microscope (obtained very $0.4 \mathrm{~s}$ ) during sliding test at $7.85 \mathrm{~mm} / \mathrm{s}$. Above a film thickness of $200 \mathrm{~nm}$, light intensity is approximately proportional to liquid film thickness [24]. Therefore, dark regions depict a) small gap thickness due to contact between specimens or b) bubbles of $\mathrm{CO}_{2}$. Axially-symmetric intensity gradients are due to gap thickness increasing away from the loading zone. 


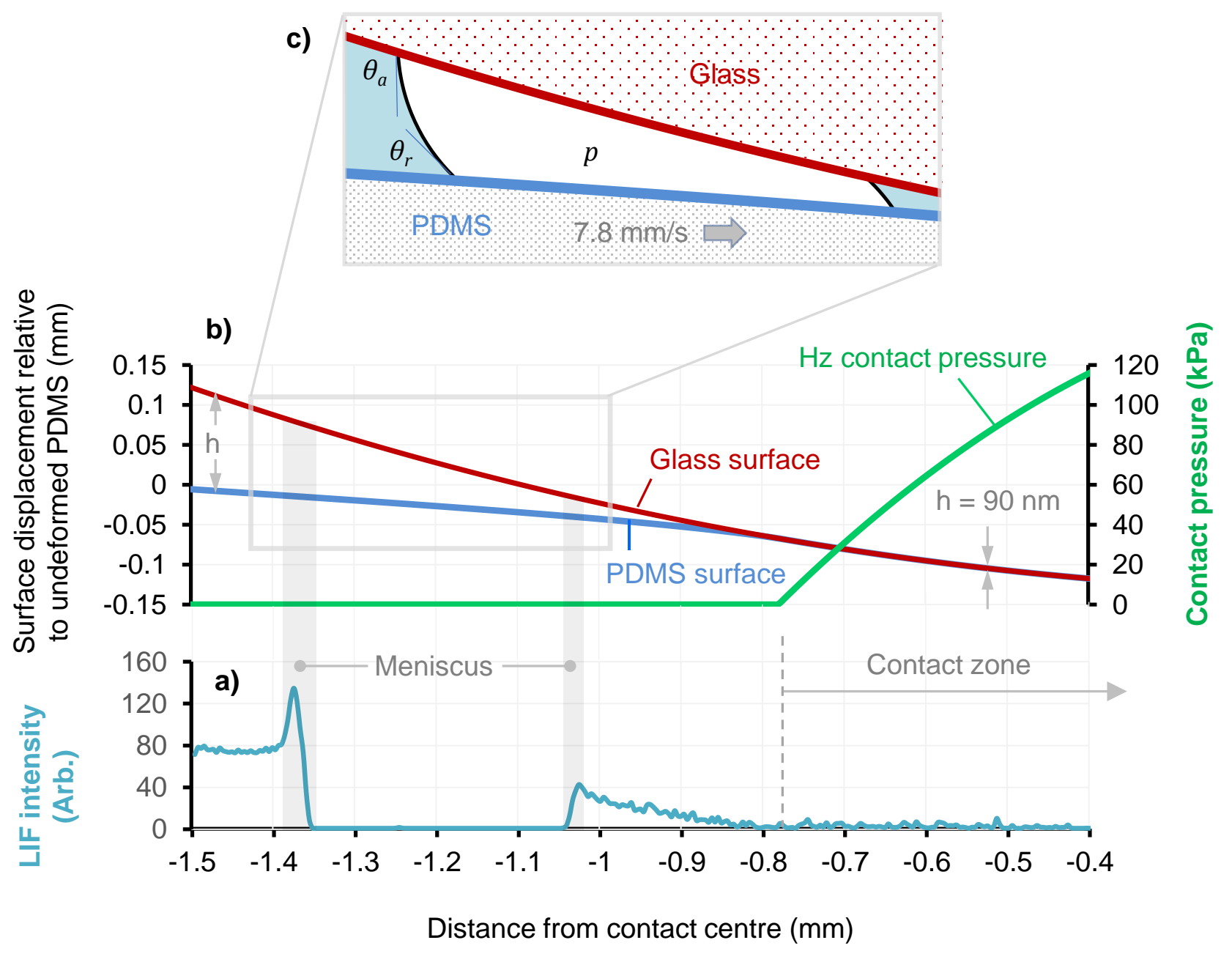

FIGURE 7 - a) LIF intensity vs. distance (taken along dashed line in Figure 6g), b) calculated pin and disc surface geometry (red and blue respectively), Hertz contact pressure (green), c) detailed image of bubble geometry (note $x \& y$ axes scales are equal).

Prior to testing, beverage consumption is mimicked by pouring the dyed, supersaturated carbonated water onto the PMDS specimen so that heterogeneous nucleation occurs at imperfections in the PDMS and any fibres present on the surface [34]. It can be seen from the videos that the motion of the PDMS entrains these surface bubbles towards to contact. Bubbles also appear to nucleate directly at the contact inlet, which we suggest is due to microbubbles (with radius less than critical value of $\sim 200 \mathrm{~nm}$ so that expansion is constrained by surface tension [34]) on the PDMS surface being obstructed by the $25 \mathrm{~nm}$ gap and increased contact pressure, as discussed below. It is also possible that frictional heating is involved in the apparent bubble nucleation around the inlet due to an increase in temperature and hence a reduction in solubility (reducing Henry's Law constant [35]). However, when the effect of friction heating is estimated as described in [36], the resulting increase in surface temperature is found to be only $\sim 0.15^{\circ} \mathrm{C}$ (see Supporting Information 2.3), which is not surprising given the low friction and low sliding speed. This is insufficient 
to alter $\mathrm{CO}_{2}$ solubility appreciably and therefore, the effects of frictional heating can be ignored.

Once bubbles approach the contact, two phenomena are observed in Figure 6: a) bubble translation and b) bubble growth. These are clearly coupled but are addressed separately in the subsequent analysis in order to simplify matters and be able to draw conclusions. First, bubble translation is considered. A number of forces act on the bubble:

$$
\begin{gathered}
\sum F=F_{\text {Surface_adhesion }}+F_{\text {Viscous_drag }}+F_{\text {Pressure_drag }}+F_{\text {contact_line_drag }} \\
+F_{\text {Confinment_gradient }}+F_{\text {Bouyancy }}
\end{gathered}
$$

The sign of each of these forces is considered positive with respect to the flow direction, therefore when the sum of forces is negative the bubble is expected to move upstream and vice versa. Considering each of the forces:

- $\quad F_{\text {Surface_Adhesion }}$ is the traction exerted by the sliding PDMS surface on the contact line. This is a complex function as the contact angle varies around the bubbles' circumference but can be estimated, according to $[33,37,38]$ from $F_{\text {Surface Adhesion }} \approx$ $2 l \gamma\left(\cos \theta_{r}-\cos \theta_{a}\right)$ where $l$ is the diameter of the bubble, $\gamma$ is the surface tension and $\theta_{a}$ and $\theta_{r}$ are the advancing and receding contact angles respectively. For the bubble geometry in Figure 7, this force is $\sim 40 \mu \mathrm{N}$ and, due to the sliding motion, it always acts in the positive/downstream direction (see Supporting Information 2.4).

- $\boldsymbol{F}_{\text {Viscous_drag }}$ is the viscous drag experienced by the bubble due to the flow of liquid between the specimens. This could be estimated [39] but is considered negligible compared to viscous forces due to the very low $\mathrm{Ca}$ [33]. Outside contact path, this force acts in the positive/downstream direction, whereas inside the contact path it acts in the negative/upstream direction due to recirculating flow. Moreover, recirculating (upstream) flow is prevalent in sphere-on-flat geometries such as this as liquid is rejected from increasingly thin gap [40])

- Fressure_drag results from pressure gradient around the bubble. Since bubble thickness is much smaller than width, $\left(h \ll 2 R_{2}\right)$, this can be estimated from $F_{\text {Pressure_drag }} \approx \Delta p \times h \times 2 R_{2}$. Approaching the contact, the pressure difference, $\Delta p$, between front and rear of bubble increases significantly as a result of the hydrodynamic pressure generated in the contact area so that this force outweighs the downstream forces and hence provides a barrier to bubbles entering the contact region. The magnitude of the hydrodynamic pressure within the contact region which provides barrier to bubble entrainment is of the same order as the calculated Hertz pressure (green line in Figure 7). For the bubble geometry in Figure 7, and a hydrodynamic pressure increase of $10 \mathrm{kPa}$, this force is estimated at $\sim 600 \mu \mathrm{N}$ (See Supporting Information 2.5). 
There is also a force caused by the difference in Laplace pressure between the front and rear bubble menisci - each with a different radius of curvature due to the changing gap height [39]. $\Delta p=\gamma\left(1 / R_{1 \_ \text {front }}-1 / R_{1}\right.$ back $)$, here $R_{1 \_ \text {front }}$ and $R_{1 \_ \text {back }}$ are the front and rear meniscus radius, which can be found geometrically [41] and which differ due the changing gap. For the bubble geometry in Figure 7, this approach suggests a force of around $\sim 75 \mu \mathrm{N}$ (see Supporting Information 2.6) directed in the upstream direction (due to the non-wetting nature of the bubble in a gap that converges in the direction of flow). Because of its dependence on the reciprocal of the gap thickness, this resistive force increases by two orders of magnitude as the $25 \mathrm{~nm}$ central film thickness is approached.

- F Confinment_gradient results from the non-parallel geometry of the upper and lower surfaces bounding the bubble. If the bubble is considered a non-wetting body, with the water preferentially wetting the surfaces, then capillary energy is reduced when the combined areas of the bubble roof and floor is reduced and vice versa. As considered by [39], for a given bubble volume, $V$, the roof and floor area, $A$, will reduce if a confined bubble moves in the direction of increasing gap height and therefore results in a force driving the bubble out of the wedge. This can be estimated from volume conservation, $A=2 \mathrm{~V} / \mathrm{h}(\mathrm{x})$, and differentiating the capillary energy due to dewetting, $\gamma A$, with respect to distance: $F_{\text {Confinement_gradient }}=2 V \gamma \frac{d}{d x}(h(x))$. A negative thickness gradient (as occurs upstream of the contact inlet) therefore results in a force away pushing the bubble back from the contact inlet. For the bubble geometry in Figure 7, this force is estimated at $\sim 20 \mu \mathrm{N}$ (see Supporting Information 2.7).

- $F_{\text {Bouyancy }}$ is negligible due to very low Bond number. Due to the downwards sloping gap at the inlet due to indentation, a component of this force is directed out of the contact.

Comparing the magnitudes, directions and gradients of these forces reveals that, in the contact path where the gap converges down to $25 \mathrm{~nm}$, bubbles are forced to translate upstream. This is because the surface drag from the sliding which acts to entrain bubbles is outweighed by upstream forces caused by the converging gap. Moreover, the latter increases rapidly as the tiny $\sim 25 \mathrm{~nm}$ thick hydrodynamic film is approached. Conversely, the forces directed upstream become negligible at large gap heights and are directed downstream when the gap diverges. This explains bubble motion observations $i$, ii, iv above. The barrier provided by the contact also explains why bubbles smaller than the critical radius are prevented from entering and thus explains the apparent nucleation. Once bubbles pass the minimum gap where the surfaces diverge, all forces become aligned in the downstream direction, which explains the rapid disappearance of bubbles such as bubble $\mathrm{A}$ (observation v). 
Considering bubble growth, the number of moles, $n$, increases within the bubble as $\mathrm{CO}_{2}$ dissolved in the water diffuses through the walls [42]. This causes bubble volume, $V$, to increase according to $V=n R T / p$ so that the pressure inside the bubble balances with the sum of the atmospheric pressure and Laplace pressure. This increase in bubble volume is facilitated by the contact line moving in the diverging gap direction since this increases the meniscus radius and reduces pressure (as opposed to expanding in the converging direction, which would lead to a reduction in radius and increase in pressure). This explains observations iv) a that, in upstream of the contact inlet, bubbles elongate in the outwards radial direction.

Bubble elongation occurs as bubbles travel downstream. In order to conserve volume, the horizontal area must increase to compensate for the reducing gap height in the flow direction. This is because as the volume is conserved (or increased to due additional $\mathrm{CO}_{2}$ gas produced), the plan view area must increase as the height reduces towards the contact. This explains the rapid lengthening of bubble $\mathrm{B}$ (observation $\mathrm{i}$ ) b).

Even though the mechanisms described above prevent bubbles progressing through the contact region, the fluid film that separates surfaces and alleviates friction is impacted nonetheless, since the flow of liquid towards the inlet and hence the generation of hydrodynamic pressure is inhibited. In fact, this bubble entrapment prolongs and compounds starvation. Numerical simulations of EHL film thickness with varying boundary conditions show that the critical distance from the meniscus to the contact inlet below which starvation occurs can be calculated from [43-45] critical distance = $a \times\left(1+3.06\left(\left(h_{c} / a\right) \times(R / a)^{2}\right)^{0.58}\right)$, where $h_{c}$ is the central film thickness $(25 \mathrm{~nm}), R$ is the glass radius $(4.5 \mathrm{~mm})$ and $a$ is the contact semi-width according to Hertz $(0.78 \mathrm{~mm})$. For the test in Figure 6, the critical distance is $1.51 \mathrm{~mm}$. This shows that bubbles reach sufficiently close to impact film thickness and provides an explanation for the increased friction shown in Figure 4. That is, bubbles reduce liquid entrainment and hence reduce hydrodynamic fluid pressure generation, causing surfaces to move closer together increasing solid-solid contact and increasing friction in the mixed regime. Moreover, this increase in solid-solid contact provides a physical barrier that further obstructs the passage of bubbles through the contact inlet.

This starvation mechanism is only effective at intermediate speeds $(3-15 \mathrm{~mm} / \mathrm{s})$, (see Figure 4), since at low speed surfaces are in contact anyway as there is no hydrodynamic film generated. At high speed, recirculation effects prevent bubbles from gaining traction at the inlet, due to - similar behaviour observed by when investigating the entrainment of wear debris particles into contacts [46]. It should be noted that this range of slide speeds, where $\mathrm{CO}_{2}$ starvation affects friction, is representative of those in the mouth during beverage consumption. 


\subsection{Mechanism 2: Protein boundary film removal}

A second set of LIF tests were run to study the effects of carbonated water on the salivary pellicle, which can be considered as an adhered surface layer (i.e. boundary film) [47] as opposed to a hydrodynamically generated film as studied in the previous section. Prior to tests where carbonated and non-carbonated liquids were entrained in the contact, the buildup of a pure salivary film was first studied. Figure 8 shows the evolution of the average LIF intensity (approximately proportional to dyed film thickness [21]) in the contact (the pixel values were averaged over a selected region within the contact area - insert Figure 8) during three steps: loading, sliding, and stationary. During the first $10 \mathrm{~s}$ of the test, specimens were pressed together to a load of $0.2 \mathrm{~N}$ statically. Dye-tagged saliva proteins are squeezed out during this step, but a portion remain trapped the contact producing an intensity of $\sim 11,600$ Arb. Sliding commences and during the next $210 \mathrm{~s}$, fluorescence intensity gradually increases to $\sim 12,500$ Arb due to the entrainment of proteins. Here, the contact is sliding at a constant speed of $0.63 \mathrm{~mm} / \mathrm{s}$ and the observed fluctuations result from the heterogeneous nature of saliva being entrained (see example of saliva agglomeration passing through the contact in Figure 8 inset). From the moment the sliding motion is halted (230 seconds), the intensity gradually reduces from $\sim 12750$ towards its initial value of $\sim 11500$, as the liquid portion of the film is squeezed out from between the surfaces. Assuming the fluorescence intensity is approximately proportional to film thickness [24], the ratio of sliding intensity to static intensity ((12750-11500)/12750) predicts that, at a sliding speed of $0.63 \mathrm{~mm} / \mathrm{s},<10 \%$ of the film thickness can be attributed to a liquid, hydrodynamic film. The remaining $\sim 90 \%$ of the film is the adhered protein boundary, which is attracted to the charged $-\mathrm{O}-\mathrm{Si}\left(\mathrm{CH}_{3}\right)_{2-}$ bonds on the PDMS surface [48]. 


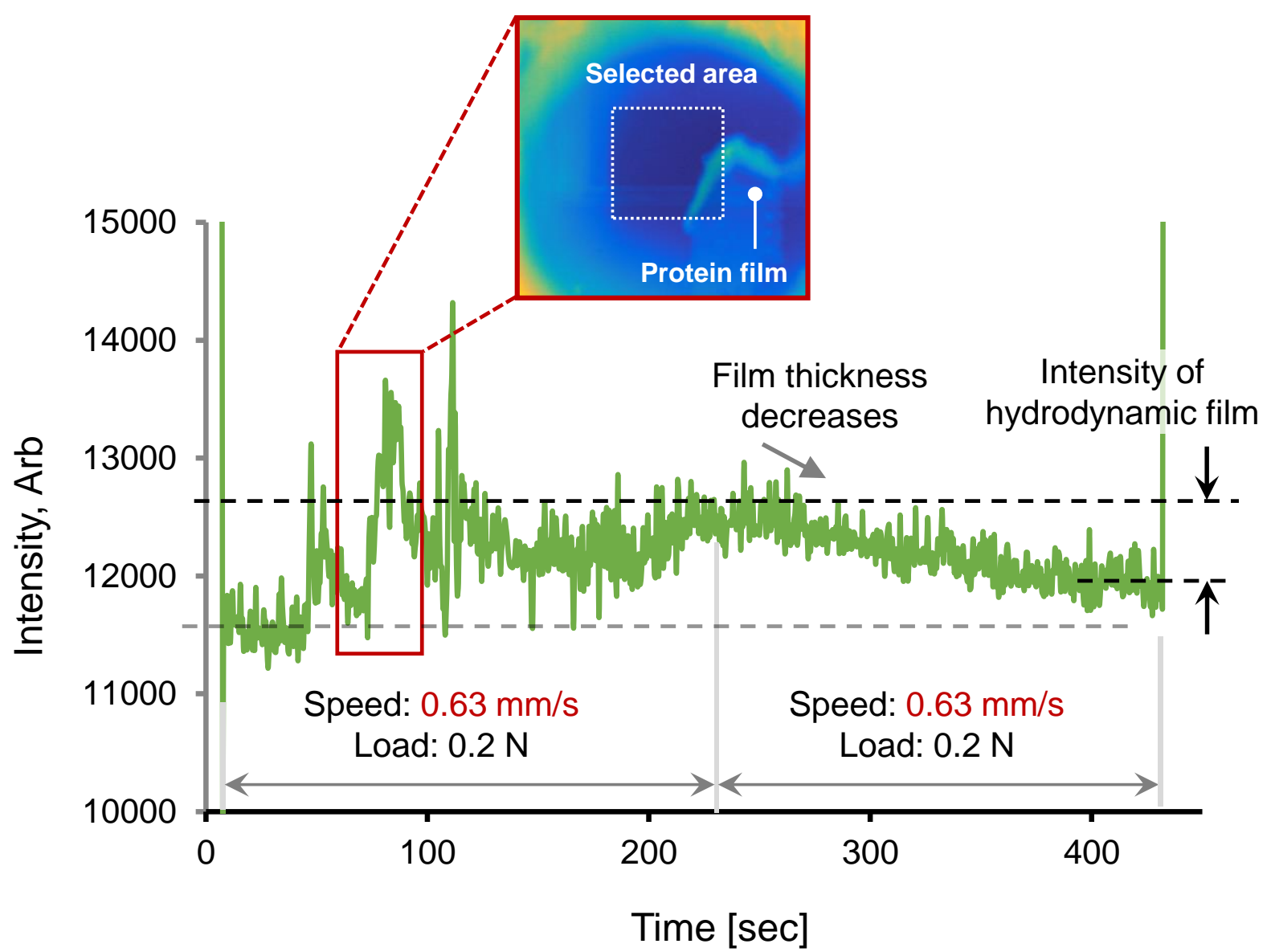

FIGURE 8 - In-contact fluorescence intensity versus time for dye-tagged saliva lubricated contact. Inset: LIF image captured at $81 \mathrm{~s}$.

Simulating the process of salivary film degradation during beverage consumption, Figure 9 shows the simultaneous variation in fluorescence intensity and friction for a test in which water (carbonated and non-carbonated) is introduced into the PDMS-glass contact lubricated with dye-tagged saliva after 10 minutes of sliding. The neat saliva friction coefficient value before adding water $(\sim 0.1)$ is considerably lower than that for pure water ( 1.2 shown in Figure 4), both measured at the same speed. This highlights the lubricious nature of the salivary film formed, and the simultaneous reduction in LIF intensity and increase in friction that occurs when water is added signals its removal. It can also be observed that the introduction of carbonated water disrupts the salivary film and subsequently increases friction to a greater extent than the pure water does (the modest film degradation following dilution with pure water can be attributed to a reduction in ionic strength [49]). Importantly, carbonated water induced reduction in film thickness of $\sim 80 \%$, is greater than the thickness of the hydrodynamic water film (see Figure 8), which proves that the adhered salivary film is actually being removed rather than just the hydrodynamic film being diluted. Further images of LIF salivary breakdown are provide in the Supporting Information. Note: additional repeat film breakdown tests were run under constant conditions and the variability was shown to be significantly less than variation between carbonate and non-carbonated samples. 


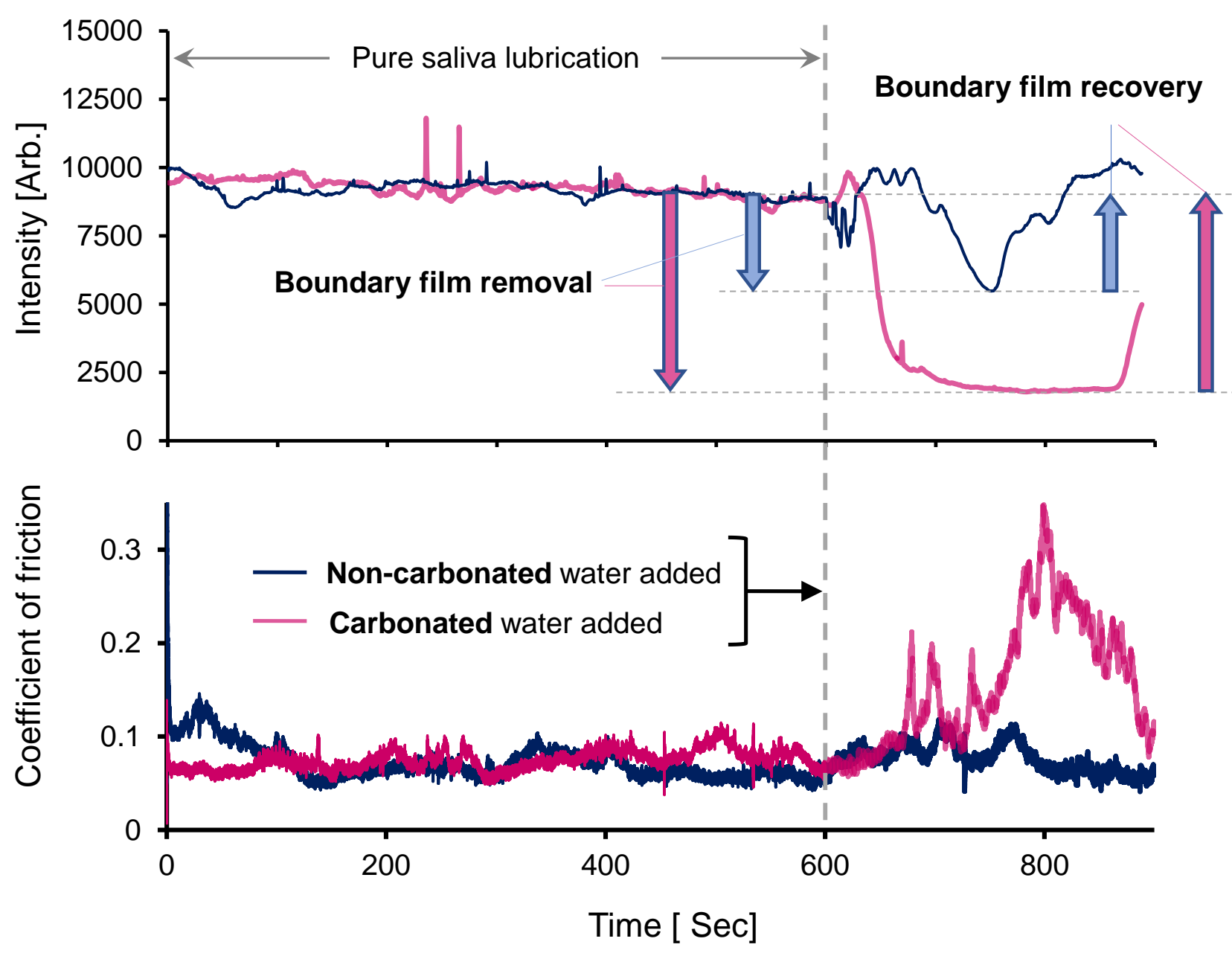

FIGURE 9 - In-contact fluorescence intensity and friction versus time for dye-tagged saliva lubricated contact, showing effects of addition of deionised water (carbonated and noncarbonated). The sliding speed for both tests is $0.63 \mathrm{~mm} / \mathrm{s}$.

By causing salivary film breakdown, carbonated water may be considered as eliciting a similar mouthfeel mechanism to that associated with astringency [50]. However, the film breakdown causing astringency is believed to be caused by polyphenols (tannins) in foods and beverages, which cross link proteins causing them to aggregated and loose surface adhesion [51], whereas in the case of carbonation it is likely caused by the presence of carbonic acid $\left(\mathrm{H}_{2} \mathrm{CO}_{3} \mathrm{pH} \sim 4\right.$, present in carbonated water in equilibrium with $\mathrm{CO}_{2}+\mathrm{H}_{2} \mathrm{O}$, and catalysed by carbonic anhydrase enzyme in saliva). This may occur either as an interaction directly between $\mathrm{H}_{2} \mathrm{CO}_{3}$ and salivary proteins or due to competition for the surface (i.e. $\mathrm{H}_{2} \mathrm{CO}_{3}$ reacting with the repeating, charged $-\mathrm{O}-\mathrm{Si}\left(\mathrm{CH}_{3}\right)_{2}$ - groups on the PDMS) thus inhibiting the hydrophobic electrostatic attraction of the salivary proteins and aiding their removal by the applied shear. It is also possible that mechanical effects, linked to the starvation mechanism discussed in the previous section, may play a role, however no such behaviour was observed in the LIF images.

\section{Conditions within the mouth}


The contact inlet is shown to be a favourable location for bubbles to nucleate and grow, however the hydrodynamic pressure and the Laplace pressure caused by the rapidly converging gap prevent their entrainment through the contact. Trapped bubbles then obstruct flow, reduce film thickness and increase friction. In the presence of saliva, carbonation also increases friction through protein removal. As friction is believed to affect mouthfeel and hence flavour perception [15,16], these results may be important in understanding carbonated taste effects - such as the perceived sweetness of flat cola (Figure 3). However, the differences between our experimental conditions and those within the mouth must be considered in order to properly understand the implications of the test results. For instance, our experiments in Section 3.1 revealing the bubble starvation mechanism involved only carbonated and non-carbonated water as the test fluid without any saliva being present. Since salivary proteins are capable of acting as surfactants, their presence would alter the surface tension and would hence affect the observed behaviour. However, this is suggested as being a secondary effect, which would not change the mechanisms in play, since surface tension and contact angle does not affect the hydrodynamic pressure drag which is the dominant factor preventing the entrainment of bubble and causing the observed contact starvation.

The applied load and range of sliding speeds used in this study are representative of those in the mouth and the PDMS elastomer is similar to the tongue in its ability to bind saliva proteins [21]. The tongue's surface is rougher than the experimental specimens (650 vs 20 $n m R_{a}[21]$ ), however, this has a reduced effect on lubrication behaviour as the latter is more compliant ( $\mathrm{E}$ $3.5 \mathrm{vs} 2300 \mathrm{kPa}$ [21]) and therefore smoothens under the applied load. For these reasons, the experimental setup used here operates under the same lubrication regime as the tongue under both water and saliva immersion [21]. The main discrepancies between experimental conditions and actuality arise due to the underformed roughness outside the loaded zone (i.e. where roughness has not been reduced by conforming to the upper surface) and thermal effects (in our tests both liquid and surfaces were at the same temperature). Both of these factors should enhance bubble formation and entrainment between surfaces in the mouth, since roughness providing nucleation sites and the difference in temperature between cooler beverage and warmer mouth cause a substantial reduction in solubility of $\mathrm{CO}_{2}$. It should also be noted that the roughness of the tongue is in part due to the presence of taste buds contained within fungiform papillae [52], whose sensitivity to detecting flavours is likely to be modulated by $\mathrm{CO}_{2}$ inducing both contact starvation and salivary film removal, as we have observed. It may explain why it is important to balance acidity and sweetener in determining the refreshment of carbonated beverages [53].

\section{Conclusions}


We assessed the effects of carbonation for the first time from a tribological point of view using a sliding interface simulating tongue/palate. Two new mechanisms are observed:

- Bubbles nucleate in the contact inlet, probably due to the converging geometry trapping microbubbles in combination with an increase in temperature. However, the hydrodynamic pressure, converging gap and surface contact prevents the bubbles from entering into the loaded zone. Bubbles thus remain trapped and grow due to diffusion, and their presence prevents fluid entering to support the surfaces. This results in more surface contact and higher friction.

- Carbonated water is more effective than non-carbonated water in removing the salivary pellicle, as shown by tests simulating beverage consumption. We suggest this is caused by $\mathrm{pH}$ rather than mechanical effects. Due to the highly lubricious nature of the salivary pellicle, its removal results in a substantial increase in friction.

These findings do not contradict the established view that carbonation elicits a response from chemical receptors but they do suggest that via complementary mechanisms other mouthfeel attributes are also affected. Not only are the observed friction-increasing mechanisms likely to contribute directly to mouthfeel, their associated surface cleaning effects should play an important role in the experience of refreshment and may also modulate the flow of tastants to the taste buds, reducing the perception of sweetness in colas for example. This would also provide an alternative explanation as to why flat colas taste sweeter. The test method combining friction and fluorescence microscopy measurements on an oral mimic is shown to be an effective means of probing beveragemouth interactions and will be used in future to study the behaviours of different food and beverage constituents.

\section{Acknowledgements}

S.K. Baier is employed by PepsiCo, Inc. The authors also acknowledge the support of PepsiCo 3255210. The views expressed in this research article are those of the authors and do not necessarily reflect the position or policy of PepsiCo, Inc.

\section{References}

[1] S.M. Kappes, S.J. Schmidt, S.Y. Lee, Relationship between physical properties and sensory attributes of carbonated beverages. Journal of Food Science 72 (2007) 1:S001-11.

[2] B.G. Green, Chemesthesis: pungency as a component of flavor. Trends Food Sci Technol (1996) 7:415-20.

[3] S. Meyer, W.E. Riha, Optimizing sweetener blends for low-calorie beverages. Food Technol (2002) 56:42-5.

[4] P.R. Ashurst, The chemistry and technology of soft drinks and fruit juices. Boca Raton, Fla.: Sheffield Academic Press (1998) p 258.

[5] C.G.J. Bisperink, A. Prins, Bubble growth in carbonated liquids, Colloids Surfaces A Physicochem. Eng. Asp. 85 (1994) 237-253. doi:10.1016/0927-7757(94)02744-7.

[6] G. Liger-Belair, The Physics and Chemistry behind the Bubbling Properties of Champagne and Sparkling Wines: A State-of-the-Art Review, J. Agric. Food Chem. 53 (2005) 2788-2802. doi:10.1021/jf048259e.

[7] C.P. Des Gachons, J. Avrillier, M. Gleason, L. Algarra, S. Zhang, E. Mura, H. Nagai, P.A. Breslin, Oral cooling and carbonation increase the perception of drinking and thirst quenching in thirsty adults. PloS One (2016) 11(9). 
[8] G.F. Woodroof, Jasper Guy Phillips, Beverages: carbonated and noncarbonated, Avi Publishing Co., Westport, CT, 1981.

[9] R.A. Clark, L. Hewson, F. Bealin-kelly, J. Hort, The Interactions of CO2, Ethanol, Hop Acids and Sweetener on Flavour Perception in a Model Beer, Chemosens. Percept. 4 (2011) 42-54. doi:10.1007/s12078-011-9087-3.

[10] J.M. Dessirier, C.T. Simons, M.I. Carstens, M. O'Mahony, E. Carstens, Psychophysical and neurobiological evidence that the oral sensation elicited by carbonated water is of chemogenic origin., Chem. Senses. 25 (2000) 277-284. doi:10.1093/chemse/25.3.277.

[11] J.M. Dessirier, C.T. Simons, M. O'Mahony, E. Carstens, The oral sensation of carbonated water: cross-desensitization by capsaicin and potentiation by amiloride, Chem. Senses. 26 (2001) 639-43. http://www.ncbi.nlm.nih.gov/pubmed/11473929.

[12] E. Carstens, M. Iodi Carstens, J.M. Dessirier, M. O'Mahony, C.T. Simons, M. Sudo, et al., It hurts so good: Oral irritation by spices and carbonated drinks and the underlying neural mechanisms, Food Qual. Prefer. 13 (2002) 431-443. doi:10.1016/S0950-3293(01)00067-2.

[13] J. Chandrashekar, D. Yarmolinsky, L. von Buchholtz, Y. Oka, W. Sly, N.J.P. Ryba, C.S. Zuker, The Taste of Carbonation, Science 326 (2009), 5951:443-445. doi:10.1126/science.1174601.

[14] M. Komai, B.P. Bryant, Acetazolamide specifically inhibits lingual trigeminal nerve responses to carbon dioxide, Brain Res. 612 (1993) 122-129. doi:10.1016/0006-8993(93)91652-9.

[15] J.R. Stokes, M.W. Boehm, S.K. Baier, Oral processing, texture and mouthfeel: From rheology to tribology and beyond, Curr. Opin. Colloid Interface Sci. 18 (2013) 349-359. doi:10.1016/j.cocis.2013.04.010.

[16] G.A. Davies, E. Wantling, J.R. Stokes, The influence of beverages on the stimulation and viscoelasticity of saliva: Relationship to mouthfeel?, Food Hydrocoll. 23 (2009) 2261-2269. doi:10.1016/j.foodhyd.2009.06.002.

[17] J.R. Stokes, 12 "Oral” tribology, in: J. Chen, L. Engelen (Eds.), Food Oral Process. Fundam. Eat. Sens. Percept., Wiley-Blackwell, 2012: pp. 265-87.

[18] J. Chen, J.R. Stokes, Rheology and tribology: Two distinctive regimes of food texture sensation, Trends Food Sci. Technol. 25 (2012) 4-12. doi:10.1016/j.tifs.2011.11.006.

[19] H.L. Gibbins, G.B. Proctor, G.E. Yakubov, S. Wilson, G.H. Carpenter, Concentration of salivary protective proteins within the bound oral mucosal pellicle, Oral Dis. 20 (2014) 707-713. doi:10.1111/odi.12194.

[20] Carpenter Guy, 3 Role of Saliva in the Oral Processing of Food, in: Food Oral Process. Fundam. Eat. Sens. Percept., Jianshe Ch, Wiley-Blackwell, 2012: pp. 201245-60.

[21] G. Carpenter, S. Bozorgi, S.-C. Vladescu, A.E. Forte, C. Myant, R.V. Potineni, T. Reddyhoff, $\mathrm{S}$. Baier, A study of saliva lubrication using a compliant oral mimic, Food Hydrocolloids 92 (2019) 10-18.

[22] G.S. Barker, B. Jefferson, S.J. Judd, The control of bubble size in carbonated beverages, Chem. Eng. Sci. 57 (2002) 565-573. doi:10.1016/S0009-2509(01)00391-8.

[23] G. Liger-Belair, The Physics and Chemistry behind the Bubbling Properties of Champagne and Sparkling Wines: A State-of-the-Art Review, J. Agric. Food Chem. 53 (2005) 2788-2802. doi:10.1021/jf048259e.

[24] C. Myant, T. Reddyhoff, H. a. Spikes, Laser-induced fluorescence for film thickness mapping in pure sliding lubricated, compliant, contacts, Tribol. Int. 43 (2010) 1960-1969. doi:10.1016/j.triboint.2010.03.013.

[25] T. Reddyhoff, J.H. Choo, H. a. Spikes, R.P. Glovnea, Lubricant flow in an elastohydrodynamic contact using fluorescence, Tribol. Lett. 38 (2010) 207-215. doi:10.1007/s11249-010-9592-6.

[26] S.-C. Vlădescu, A. Ciniero, K. Tufail, A. Gangopadhyay, T. Reddyhoff, Looking into a laser textured piston ring-liner contact, Tribol. Int. 115 (2017) 140-153. doi:10.1016/j.triboint.2017.04.051.

[27] K.S. Phillips, Q. Cheng, Microfluidic Immunoassay for Bacterial Toxins with Supported Phospholipid Bilayer Membranes on Poly (dimethylsiloxane), Anal. Chem. 92521 (2003) 5243-5250.

[28] J.H.H. Bongaerts, K. Fourtouni, J.R. Stokes, Soft-tribology: Lubrication in a compliant PDMSPDMS contact, Tribol. Int. 40 (2007) 1531-1542. doi:10.1016/j.triboint.2007.01.007. 
[29] D.M. Dresselhuis, H.J. Klok, M.A.C. Stuart, R.J. De Vries, G.A. Van Aken, E.H.A. De Hoog, Tribology of $\mathrm{o} / \mathrm{w}$ emulsions under mouth-like conditions: Determinants of friction, Food Biophys. 2 (2007) 158-171. doi:10.1007/s11483-007-9040-9.

[30] J. de Vicente, J.R. Stokes, H.A. Spikes, The frictional properties of Newtonian fluids in rolling - Sliding soft-EHL contact, Tribol. Lett. 20 (2005) 273-286. doi:10.1007/s11249-005-9067-3.

[31] M. Esfahanian, B.J. Hamrock, Fluid-film lubrication regimes revisited, Tribology Transactions 34 (1991) 4:628-32.

[32] M. Scaraggi, G Carbone, B.N. Persson, D. Dini, Lubrication in soft rough contacts: A novel homogenized approach. Part I-Theory. Soft Matter. 7(21) 2011:10395-406.

[33] B. Blackmore, D. Li, J. Gao, Detachment of bubbles in slit microchannels by shearing flow. Journal of colloid and interface science 241 (2001) 2:514-20.

[34] M. W. Carr, A. R. Hillman, S. D. Lubetkin, Nucleation rate dispersion in bubble evolution kinetics J. Colloid Interface Sci. 169 (1995) 135-142.

[35] G. Liger-Belair, The physics behind the fizz in champagne and sparkling wines. The European Physical Journal Special Topics. 201 (2012) 1:1-88.

[36] T. Reddyhoff, A. Schmidt, and H. A. Spikes, 2019. Thermal conductivity and flash temperature. Tribology Letters, 67(1), p.22.

[37] J.Y. Leong, J. Zhang, S.K. Sinha, A. Holmes, H. Spikes, T. Reddyhoff, Confining liquids on silicon surfaces to lubricate MEMS. Tribology Letters. 59 (2015) 1:15.

[38] M. Mahe, M. Vignes-Adler, A. Rousseau, C.G. Jacquin, P.M. Adler, Adhesion of droplets on a solid wall and detachment by a shear flow: I. Pure systems. Journal of colloid and interface science. 126 (1988) 1:314-28.

[39] E. Reyssat, Drops and bubbles in wedges. Journal of fluid mechanics (2014) 748:641-62.

[40] Shieh, J. and Hamrock, B. J., Film Collapse in EHL and Micro-EHL, ASME J. Trib., 113. (1991) 372-377.

[41] C. Luo, M. Mrinal, X. Wang, Self-propulsion of Leidenfrost Drops between Non-Parallel Structures. Scientific reports. 7 (2017) 1:1-2.

[42] P. Peñas-López, B. van Elburg, M.A. Parrales, J. Rodríguez-Rodríguez, Diffusion of dissolved $\mathrm{CO}_{2}$ in water propagating from a cylindrical bubble in a horizontal Hele-Shaw cell, Physical Review Fluids, 2 (2017) 6:063602.

[43] B.J. Hamrock, D. Dowson, Isothermal elastohydrodynamic lubrication of point contacts: Part IV-starvation results, J. of Lubrication Tech. 99 (1977) 1: 15-23.

[44] L.D. Wedeven, D. Evans, A. Cameron, Optical analysis of ball bearing starvation ASME J Lubr Technol, 93 (1971) 349-361

[45] J. Hili, C. Pelletier, L. Jacobs, A. Olver, T. Reddyhoff, High-Speed Elastohydrodynamic Lubrication by a Dilute Oil-in-Water Emulsion. Tribology Transactions 61 (2018) 2:287-94.

[46] R.S. Dwyer-Joyce, J. Heymer, The Entrainment of Solid Particles into Rolling Elastohydrodynamic Contacts, Tribol. Ser. 1996 (31AD) 135.140.

[47] C. Hannig, M. Hannig, A. Kensche, G. Carpenter, The mucosal pellicle-An underestimated factor in oral physiology, Archives of Oral Biology 80 (2017) 144-152.

[48] K.S. Phillips, Q. Cheng, Microfluidic immunoassay for bacterial toxins with supported phospholipid bilayer membranes on poly (dimethylsiloxane). Analytical chemistry 77 (2005) 1:327-334

[49] L. Macakova, G.E. Yakubov, M.A. Plunkett, J.R. Stokes, Influence of ionic strength on the tribological properties of pre-adsorbed salivary films. Tribology International, 44(9), (2011) pp.956-962.

[50] S. Ma, H. Lee, Y. Liang, F. Zhou, Astringent mouthfeel as a consequence of lubrication failure, Angewandte Chemie International Edition 55 (2016) 19:5793-7.

[51] T. Ozawa, T.H. Lilley, E. Haslam, Polyphenol interactions: astringency and the loss of astringency in ripening fruit, Phytochemistry 26 (1987) 11:2937-42.

[52] A. Gardner, G. Carpenter, Anatomical stability of human fungiform papillae and relationship with oral perception measured by salivary response and intensity rating, Scientific Reports 9 (2019) 9759. 
[53] J. Scott, Sugar its role in soft drinks, Soft Drinks Manage Int. (1988) 24-6.

\section{Supporting Information}

\section{Calculation of film thickness}

Central film thickness in an iso-viscous elastohydrodynamic contact can be calculated according to Esfahanian and Hamrock .

$$
h_{c}=3.2 R^{0.76}(\eta U)^{0.66} W^{-0.21} E^{*-0.45}=25.0 \mathrm{~nm}
$$

where:

- $R=0.045 \mathrm{~m}$ is the radius of contact.

- $\eta=0.0010016$ Pas is the viscosity of water

- $U=0.003925 \mathrm{~ms}^{-1}$ is the average surface sliding speed

- $W=0.2 \mathrm{~N}$ is the applied load

- $E^{*}=\left(\left(\frac{1-v_{1}^{2}}{E_{1}}\right)+\left(\frac{1-v_{2}^{2}}{E_{2}}\right)\right)^{-1}=2.846 \mathrm{MPa}$

- $E_{1}=2.270 \times 10^{6} \mathrm{~Pa}$ is Young's modulus of PDMS (lower specimen)

- $v_{1}=0.45$ is the Poisson's ratio of PDMS (lower specimen)

- $E_{2}=75 \times 10^{9} \mathrm{~Pa}$ is Young's modulus of glass (upper specimen)

- $v_{2}=0.22$ is the Poisson's ratio of glass (upper specimen)

\section{Calculation of contact pressure}

Maximum contact pressure can be calculated according to Hertz:

$$
P_{0}=\frac{3}{2} P_{m}=157.0 \mathrm{MPa}
$$

where $P_{m}=\left(\frac{3 W R}{2 E^{*}}\right)^{1 / 3}=104.6 \mathrm{MPa}$ is the mean contact pressure.

\section{Estimation of frictional heating effects}

The increase in temperature due frictional heating in the contact can be calculated as described in [1]:

Frictional heat generated per second:

$$
\dot{Q}=\mu W U_{s}=0.16 \mathrm{~mW}
$$

where:

- $\mu=0.1 \mathrm{~m}$ is the coefficient of friction for saliva lubricated interface (measured from Figure 9). 
- $W=0.2 \mathrm{~N}$ is the applied load

- $U_{s}=0.00785 \mathrm{~m} / \mathrm{s}$ is the sliding speed

Estimated maximum surface temperature rise:

$$
T_{\max }=\frac{3}{8} \frac{\dot{Q}}{b K}=0.15^{\circ} \mathrm{C}
$$

where:

- $b=\left(\frac{3 W R}{4 E^{*}}\right)^{\frac{1}{3}}=0.000780 m$ is the Hertz contact width

- $K=0.5 \mathrm{Wm}^{-1} \mathrm{~K}^{-1}$ is the conductivity of biological tissue [2]

\section{Calculation of surface adhesion of bubble}

$$
F_{\text {surface-adhesion }} \approx 2 l \gamma\left(\cos \theta_{r}-\cos \theta_{a}\right)=41.6 \mu \mathrm{N}
$$

where

- $l=0.00035 \mathrm{~m}$ is the length of bubble (measured from Figure 7).

- $\gamma=0.0728 \mathrm{~N} / \mathrm{m}$ is the surface tension of water

- $\theta a=100^{\circ}$ is the advancing contact angle (water on PDMS)

- $\theta_{r}=50^{\circ}$ is the receding contact angle (water on PDMS)

\section{Calculation of pressure drag on bubble}

$$
F_{\text {Pressure-drag }}=\Delta p h \times 2 R_{2}=600 \mu N
$$

where:

- $\Delta p=10000 \mathrm{~Pa}$ is the hydrodynamic pressure (measured from Figure 7)

- $h=0.001 \mathrm{~m}$ is the bubble height (measured from Figure 7)

- $2 R_{2}=0.0003 \mathrm{~m}$ is the bubble width (measured from Figure 7)

\section{Calculation of Laplace pressure force}

$$
\Delta p=\gamma\left(1 / R_{1-\text { front }}-1 / R_{1-\text { back }}\right)=76.4 \mu \mathrm{N}
$$

where:

- $R_{1-f r o n t}=0.000574 \mathrm{~m}$ is the radius of front of bubble (measured from Figure 7)

- $R_{1}$ back $=2.72 \times 10^{-5}$ is the radius of back of bubble (measured from Figure 7)

- $\gamma=0.0728 \mathrm{~N} / \mathrm{m}$ is the surface tension of water 
$F_{\text {Confinement_gradient }}=2 V \gamma \frac{d}{d x}(h(x))=18.2 \mu N$

where:

- $\mathrm{V}=0.000625 \mathrm{~m}^{3}$ is the bubble volume (measured from Figures 6 and 7 )

- $\frac{d}{d x}(h(x))=0.4$ is the slope of the converging gap (measured from Figure 7)

[1] Reddyhoff, T., Schmidt, A. and Spikes, H., 2019. Thermal conductivity and flash temperature. Tribology Letters, 67(1), p.22.

[2] Bhattacharya, A. and Mahajan, R.L., 2003. Temperature dependence of thermal conductivity of biological tissues. Physiological measurement, 24(3), p.769. 